Поступила в редакцию 13.03.2020

Подписана в печать 15.06.2020

\title{
МОДЕЛИРОВАНИЕ И ПАРАМЕТРИЧЕСКАЯ ОПТИМИЗАЦИЯ ПРОВЕДЕНИЯ ЭКСПЕРТНОГО ОПРОСА С ПОМОЩЬЮ SLICЕ-МАТРИЦ
}

\author{
() 2020 О. В. Пьянков, А. А. Терентьев \\ Воронежский институт МВД России \\ пр-т. Патриотов, 53, 394065 Воронеж, Российская Федерация
}

\begin{abstract}
Аннотация. Актуализируется создание и применение ситуационных центров органов внутренних дел в условиях возрастания сложности и неоднородности угроз, возникающих в современном обществе. Необходимость решения разнородных и разноплановых задач в целях обеспечения общественной безопасности и охраны общественного порядка требует привлечения специалистов-экспертов. В статье рассматривается проблема проведения и обработки результатов экспертного опроса попарного сравнения альтернатив в ситуационных центрах органов внутренних дел с применением ранее разработанного алгоритма, использующего slice-матрицы. Предлагается в качестве параметров проведения оптимизации попарного сравнения осуществлять выбор между скоростью и точностью, зависящих как от числа сравниваемых объектов одним экспертом, так и общим числом сравниваемых альтернатив. Сформулированы задачи параметрической оптимизации проведения экспертного опроса. С позиций теории информации, известных положений о количестве информации и энтропии разрабатывается подход, позволяющий учитывать информационные потери при формировании slice-матриц для отдельного эксперта. С точки зрения уменьшения числа попарных сравнений по отношению к общему числу пар альтернатив разрабатывается подход, позволяющий осуществить оценку выигрыша в скорости проведения экспертного опроса с применением slice-матриц. Делается обобщенный вывод о численном равенстве скорости и потери информации при использовании slice-матриц. Приводятся результаты вычислительного эксперимента по определению скорости и потери информации при проведении экспертного опроса с использованием slice-матриц. Дополнительно приводится пример зависимостей параметров оптимизации от общего числа сравниваемых альтернатив и размерности slice-матриц, делается вывод о выборе оптимального значения параметров проведения экспертного опроса.
\end{abstract}

Ключевые слова: ситуационный центр, количество информации, энтропия, параметрическая оптимизация.

\section{ВВЕДЕНИЕ}

В настоящее время по указанию Президента Российской Федерации создается система распределенных ситуационных центров (СРСЦ), которая является принципиально новым высокотехнологическим кластером, цель которого - обеспечить информационно-аналитическую поддержку государственного управления и повысить эффективность

Терентьев Александр Андреевич e-mail: Alextt02021993@yandex.ru принятия управленческих решений, используя сведения распределенного информационного фонда СРСЦ и иных банков данных различного объёма и уровня [1]. При этом в состав ядра СРСЦ входят ситуационные центры органов внутренних дел.

Ситуационный центр - это наукоемкий комплекс программно-технических средств обработки и отображения информации, информационно-аналитических систем и информационных ресурсов, информационно-телекоммуникационных сетей, технических и программных средств обеспечения

Контент доступен под лицензией Creative Commons Attribution 4.0 License.

The content is available under Creative Commons Attribution 4.0 License. 


\section{А. П. Попов, С. Г. Тихомиров, И. А. Хаустов, А. А. Хвостов, Г. С. Тихомиров}

необходимого уровня информационной безопасности, а также специально подготовленный персонал [2]. Учитывая масштаб решаемых задач, ситуационный центр МВД России также выполняет функции аналитического характера и ситуационного управления сложными организационно-техническими процессами, включая сопряжение различных каналов связи.

Функционирование ситуационных центров органов внутренних дел напрямую зависит от компетентности сотрудников, осуществляющую работу на всех уровнях системы. Поскольку перечень вопросов, решаемых ситуационным центром, широк и разнообразен, то установившейся практикой является привлечение экспертов, которые являются интеллектуальным потенциалом ситуационных центров. От экспертов зависит эффективность принимаемых решений, которые в дальнейшем будут влиять на действия сотрудников органов внутренних дел при исполнении своих служебных обязанностей.

\section{1. АНАЛИЗ РЕЗУЛЬТАТОВ ПРЕДШЕСТВУЮЩИХ РАБОТ}

При решении различных задач могут возникать необходимость в сравнении нескольких объектов с целью выявления наиболее важного из них, в связи с чем применяется метод попарного сравнения [3]. Под объектами в данном случае могут выступать:

- характеристики решаемых задач;

- действия, которые необходимо выполнять для обеспечения правопорядка;

- силы и средства, привлекаемые для охраны общественного порядка и т. д.

Одним из недостатков метода попарного сравнения является получение несогласованных результатов при необходимости осуществления сравнений значительного числа объектов. Эксперт может начать противоречить сам себе, если в ходе попарного сравнения ему будет предоставляться десятки или сотни объектов, что приведет к нарушению условия транзитивности. В работе [4] разработана методика, которая позволяет решить эту проблему делением всего множества объектов на подмножества, объединяемых по разным критериям (например, количественные или качественные характеристики, признаков наличия). В каждой из этих групп собрано сравнительно небольшое число однородных признаков, что существенно облегчает построение соответствующих матриц парных сравнений. Однако, разработанная методика не позволяет осуществлять попарное сравнение между собой объектов, находящихся в разных подмножествах, что может приводить к неадекватным выводам.

В связи с этим был разработан численный метод получения вектора приоритетов [5]. Суть данного алгоритма заключается, в том, что исходная матрица попарных сравнений $A$ размером $n$ разбивается на $m$ slice-матриц $B_{i}(i=1,2, \ldots, m)$ размером $N=6, \ldots 9$. Выбор размера slice-матриц определяется исходя из общих рекомендаций, позволяющих учитывать психологические особенности мышления экспертов [3]. В каждую slice-матрицу включается эталонный объект, выбранный из всего множества объектов, после чего эксперты, используя фундаментальную шкалу, предложенную Т. Саати, заполняют slice-матрицы $B_{i}$. За счет наличия в каждой slice-матрице эталонного объекта появляется возможность объединения в единый вектор приоритетов для всех рассматриваемых объектов векторов приоритетов всех slice-матриц. Стоит также добавить, что применение slice-матриц позволяет как использовать несколько экспертов (т. е. распараллелить процесс попарного сравнения всех объектов), что несомненно позволяет получить значительный выигрыш по времени, так и представлять одному эксперту множество небольших по размеру матриц, что обеспечит повышение согласованности получаемых результатов. Автоматизация заполнения и обработки slice-матриц позволяет получить вектора приоритетов и выделить наиболее важный объект $[6,7]$.

Получаемый в этом случае вектор приоритетов, по понятным причинам, будет содержать значения достаточно малые по своей величине, однако, поскольку метод попарного сравнения в первую очередь используется для сравнения между собой объектов, то абсо- 
лютно неважным является порядок величин приоритетов объектов. Другими словами абсолютно на важно, будут ли значения приоритетов равны 0,1 и 0,2 или 0,0001 и 0,0002 . Важно, ли то что объект со значением 0,0002 (как и со значением 0,2) будет иметь более высокую значимость по сравнению с объектом, имеющим значение 0,0001 (или 0,1 ).

\section{2. ПОСТАНОВКА ЗАДАЧИ}

Предложенный метод проведения попарного сравнения множества объектов за счет разделения исходной матрицы $A$ на $m$ slice-матриц $B_{i}$ и привлечения нескольких экспертов позволяет с одной стороны уменьшить время, необходимое для выполнения сравнений, однако, с другой - приводит к потери информации. Оба эти параметра находятся в противоречии друг с другом: улучшение одного неизбежно ведет к ухудшению другого.

В связи с этим, необходимо определить такие параметры slice-матриц (размерность $N$, количество матриц $m$ ), которые позволят либо минимизировать потерю информации, либо обеспечить максимальный выигрыш в скорости проведения экспертного сравнения. Таким образом, можно сформулировать следующие задачи параметрической оптимизации:

Задача 1. Минимизация потери информации П при заданной минимальной скорости $C_{\text {min }}$ :

$$
\begin{gathered}
\Pi \rightarrow \min , \\
\text { при } C \geq C_{\min } .
\end{gathered}
$$

Задача 2. Максимизация скорости проведения экспертного сравнения $C$ при заданных максимальных потерях информации $\Pi$ :

$$
\begin{gathered}
C \rightarrow \max , \\
\text { при } \Pi \leq \Pi_{\max } .
\end{gathered}
$$

\section{3. РЕШЕНИЕ ЗАДАЧИ}

Для расчёта потери информации воспользуемся методами теории информации. В качестве меры информации можно использовать любую монотонно убывающую функцию от вероятности $f(P(x))$, где $x$ - какое либо событие, $P(x)$ - его вероятность $[7,8]$. В соот- ветствии с теорией информации будем использовать понятие количество инбормачии $I(x)$, рассчитываемое согласно следующего выражения:

$$
I(x)=\log _{2}\left(\frac{1}{P(x)}\right)=-\log _{2}(P(x))
$$

Тогда, показатель собственной информации $I\left(x_{k}\right)$ - количество информации, которое несет один конкретный символ источника $x_{k}$, вычисляется в соответствии с выражением:

$$
I\left(x_{k}\right)=-\log _{2}\left(P\left(x_{k}\right)\right)>0
$$

Для вычисления среднего количество информации, которое может нести один символ источника, будем использовать понятие энтропия источника $H(X)$. Она определяется как математическое ожидание собственной информации $I\left(x_{k}\right)$.

$$
H(X)=-\sum_{k=1}^{h} P\left(x_{k}\right) \cdot \log _{2} P\left(x_{k}\right)
$$

В соответствии с 3-м свойством энтропии максимальное значение энтропии определяется только алфавитом источника $M$ и достигается при равновероятностных символах источника [8]. Будем считать, что значения парных сравнений равновероятны тогда:

$$
H(X)=\log _{2} M
$$

где $M$ - количество возможных значений попарного сравнения в одной ячейке матрицы.

Согласно фундаментальной шкале, таких значений 17: 1 - равная предпочтительность, 2 - слабая степень предпочтения, ..., 9 - абсолютное предпочтение; $1 / 2$ - обратное значение степени предпочтения $2, \ldots, 1 / 9$ - обратное значение степени предпочтения 9.

Поскольку эти значения являются равновероятными и независимыми, а каждый символ в матрице несет максимальное значение, то общее количество информации в одной матрице будет равно:

$$
I(x)=K_{c} \cdot H(X)
$$

где $K_{c}$ - количество попарных сравнений в одной матрице

$$
K_{c}=\frac{n(n-1)}{2}
$$


А. П. Попов, С. Г. Тихомиров, И. А. Хаустов, А. А. Хвостов, Г. С. Тихомиров

Для исходной матрицы $A$ :

$$
I(x)=\frac{n(n-1)}{2} \cdot \log _{2} M
$$

Поскольку количество информации $I(x)$ обладает свойством аддитивности [6], согласно которому количество информации, содержащейся в нескольких независимых сообщениях $x_{i}$, равно сумме количества информации в каждом из них, то, принимая каждую slice-матрицу как независимое сообщение, общее количество информации во всех $m$ slice-матрицах определяется выражением

$$
\begin{gathered}
I_{\Sigma}(x)=\frac{N(N-1)}{2} \cdot m \cdot \log _{2} M \\
m=1+\text { ceil }\left[\frac{n-N}{N-1}\right]+ \\
+1 \cdot \text { floor }\left[\frac{n-N-R \cdot(N-1)}{N-1}\right]
\end{gathered}
$$

где $R=$ ceil $\left[\frac{n-N}{N-1}\right]$;

ceil[] - функция, принимающая значение ближайшего меньшего целого числа, напримep, ceil $[3,5]=3$;

floor[] - функция, принимающая значение ближайшего большего целого числа, например, floor $[3,5]=4$.

Учитывая формулы нахождения количества информации (7) и (8), найдем отношение количества информации хранящейся в исходной матрице $A$ к информации хранящейся в slice-матрицах $I_{\Sigma}(x)$. Данное отношение будем называть потерей инбормации и обозначать $(\Pi)$ :

$$
\Pi=\frac{\frac{n(n-1)}{2} \cdot \log _{2} M}{m \cdot \frac{N(N-1)}{2} \cdot \log _{2} M}=\frac{n(n-1)}{m \cdot N(N-1)}
$$

Аналогично если для исходной матрицы в виду её симметричности, нам потребуется выполнить $\frac{n(n-1)}{2}$ сравнений, то для каждой slice-матрицы количество сравнений составит $\frac{N(N-1)}{2}$ раз. Учитывая, что имеется $m$ slice-матриц общее число сравнений составит $m \cdot \frac{N(N-1)}{2}$. Тогда, сравнивая оба выражения, получим выигрыш скорости равный

$$
C=\frac{n(n-1)}{2} / \frac{m N(N-1)}{2}=\frac{n \cdot(n-1)}{m \cdot N(N-1)}
$$

Данное отношение будем называть скоростью и обозначать $-C$.

Альтернативно можно использовать подходы, связанные на расчете не относительного изменения начальных и конечных значений, как это сделано в (10) и (11), а на расчете абсолютных изменений (формулы - (12), (13)), или расчете отношения абсолютных изменений к начальным значениям (формулы - (14), (15)).

$$
\begin{gathered}
\Pi_{\grave{a}}=\frac{n(n-1)}{2} \cdot \log _{2} M-m \cdot \frac{N(N-1)}{2} \cdot \log _{2} M= \\
=\frac{\log _{2} M}{2}(n(n-1)-m N(N-1)) . \\
C_{\grave{a}}=\frac{n(n-1)-m N(N-1)}{2} . \\
\Pi_{o}=\frac{\left(\frac{n(n-1)}{2} \cdot \log _{2} M-m \cdot \frac{N(N-1)}{2} \cdot \log _{2} M\right)}{\frac{n(n-1)}{2} \cdot \log _{2} M}= \\
=\frac{\Pi_{\grave{a}}}{\frac{n(n-1)}{2} \cdot \log _{2} M}=1-\frac{1}{\Pi} . \\
C_{\hat{\imath}}=\frac{n(n-1)}{2}-\frac{m N(N-1)}{2} \\
=\frac{n(n-1)-m N(N-1)}{n(n-1)}=1-\frac{1}{C} .
\end{gathered}
$$

В результате сравнения формул (10) и (11), можно сказать, что скорость заполнения slice-матриц и возможная потеря информации при этом будут числено равны $C=\Pi$.

Таким образом анализируя полученное равенство, следует вывод, что перед тем как эксперту (экспертам) приступать к заполнению slice-матриц, необходимо выбрать какой показатель $C$ или $П$ наиболее важен для текущей задачи, решаемой в ситуационном центре ОВД. Например, могут возникать задачи (оперативные, возникновение чрезвычайной ситуации и т. д.), требующие решения в наикратчайшие сроки, а могут возникать задачи, где время не играет главную роль, а главным является точность решения. 
Системный анализ и синтез предиктивной системы управления проиессом термоокислительной ...

\section{4. РЕЗУЛЬТАТЫ ИССЛЕДОВАНИЙ И ИХ ОБСУЖДЕНИЕ}

Рассмотрим пример, когда при заполнении slice-матриц экспертам важно, получить как можно точные значения при принятии решения. Таким образом, руководствуясь алгоритмом предложенным в статье [3], возможный диапазон размерности одной slice-матрицы состоит от 6 до 9. Тогда для того, чтобы минимизировать потери (10), необходимо максимизировать $N$, при $n=150$ получим:

$$
\begin{gathered}
m=1+\text { ceil }\left[\frac{n-N}{N-1}\right]+ \\
+1 \cdot \text { floor }\left[\frac{n-N-R \cdot(N-1)}{N-1}\right]= \\
=1+\text { ceil }\left[\frac{150-9}{9-1}\right]+ \\
+1 \cdot \text { floor }\left[\frac{150-9-R \cdot(N-1)}{9-1}\right]=19 \\
\Pi=C=\frac{150 \cdot(150-1)}{19 \cdot 9 \cdot(9-1)}=16,337
\end{gathered}
$$

Для остальных возможных параметров результаты представлены в табл. 1.

Таблииза 1. Значение Скорости (Потери информации) для различных $m$ и $N$

[Table 1. Speed value (information Loss)

\begin{tabular}{|c|c|c|c|c|}
\multicolumn{5}{|c|}{ for different $m$ and $N]$} \\
\hline$N$ & $m$ & $n$ & Скорость $C$ & $\begin{array}{c}\text { Потери } \\
\text { информации } \Pi\end{array}$ \\
\hline 9 & 19 & 150 & 16,3377 & 16,3377 \\
\hline 8 & 22 & 150 & 18,1412 & 18,1412 \\
\hline 7 & 25 & 150 & 21,2857 & 21,2857 \\
\hline 6 & 30 & 150 & 24,8333 & 24,8333 \\
\hline
\end{tabular}

Из табл. 1 видно, что при максимальном значении $N$ скорость заполнения и потеря информации наименьшие, а при минимальной размерности данные показатели максимальны. Согласно табл. 1 построим график Скорости (Потери информации) от количества и размерности slice-матриц (рис. 1), в целях улучшения восприятия дискретной сетки значений, изобразим её с помощью 3D поверхности.

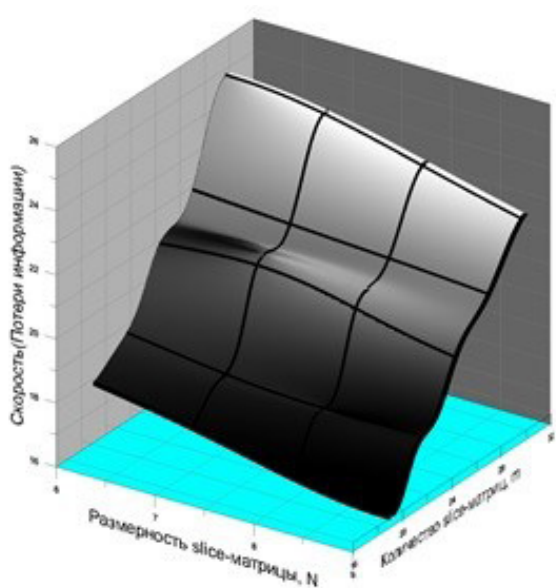

Рис. 1. Скорость (потери информации) от количества и размерности slice-матрии [Fig. 1. Speed (information Loss) of the number and dimension of slice matrices]

На рис. 1 изображена поверхность с цветовой гаммой, переходящей от темного цвета к светлому, где светлым цветом показана область максимальной потери информации и максимальной скорости при количестве slice-матриц равном 6. Темным цветом выделена область, относящая к минимальной потери информации и минимальной скорости при количестве slice-матриц равном 9. Серая область цвета показывают промежуточные значения между максимальными и минимальными значениями.

В качестве альтернативного подхода при расчете скорости заполнения slice-матриц и количества потери информации воспользуемся формулами (12), (13).

$$
\begin{gathered}
\Pi_{a}=\frac{\log _{2} 17}{2}(150 \cdot(150-1)-19 \cdot 9 \cdot(9-1))= \\
=43838,03897 \text { бит. } \\
C_{a}=\frac{150 \cdot(150-1)-19 \cdot 9 \cdot(9-1)}{2}=10725 \text { сравн. }
\end{gathered}
$$

Для остальных параметров, результаты расчетов приведены в табл. 2.

Из табл. 2 видно, что соотношение скорости заполнения slice-матриц к потери информации является прежним, как и в табл. 1. Чем выше скорость заполнения slice-матриц (т. е. чем меньше сравнений нам требуется выполнить или чем больше $C_{a}$ - разница между начальным числом сравнений и конечным), тем большее количество информации теряется. 


\section{А. П. Попов, С. Г. Тихомиров, И. А. Хаустов, А. А. Хвостов, Г. С. Тихомиров}

Таблииа 2. Отношение абсолютных значений скорости к абсолютным значениям потери инбормации

[Table 2. The ratio of the absolute values of speed to the absolute values of information loss]

\begin{tabular}{|c|c|c|c|c|}
\hline$N$ & $m$ & $n$ & $\begin{array}{c}\text { Потери ин- } \\
\text { формации } \\
\Pi_{a}, \text { бит }\end{array}$ & $\begin{array}{c}\text { Скорость } C_{a} \\
\text { сравнений }\end{array}$ \\
\hline 9 & 19 & 150 & 42881,57267 & 10491 \\
\hline 8 & 22 & 150 & 43159,52014 & 10559 \\
\hline 7 & 25 & 150 & 43531,47926 & 10650 \\
\hline 6 & 30 & 150 & 43838,03897 & 10725 \\
\hline
\end{tabular}

В заключении для подтверждении альтернативного подхода при расчете скорости заполнения и потери информации, воспользуемся формулами (14), (15).

$$
\begin{gathered}
\Pi_{o}=\frac{42881,57267}{\frac{150 \cdot(150-1)}{2} \cdot \log _{2} 17}=1-\frac{1}{16,3377}= \\
=0,938791946 . \\
C_{o}=\frac{150 \cdot(150-1)-19 \cdot 9 \cdot(9-1)}{150 \cdot(150-1)}= \\
=1-\frac{1}{16,3377}=0,938791946 .
\end{gathered}
$$

Расчеты для остальных параметров представлены в табл. 3.

Таблица 3. Отношение абсолютных изменений к начальным значениям скорости потери информации

[Table 3. The ratio of the absolute changes to the initial values of speed to the loss of information]

\begin{tabular}{|c|c|c|c|c|}
\hline$N$ & $m$ & $n$ & $\begin{array}{c}\text { Потери ин- } \\
\text { формации } \Pi_{o}\end{array}$ & Скорость $C_{o}$ \\
\hline 9 & 19 & 150 & 0,939 & 0,939 \\
\hline 8 & 22 & 150 & 0,944 & 0,944 \\
\hline 7 & 25 & 150 & 0,953 & 0,953 \\
\hline 6 & 30 & 150 & 0,960 & 0,960 \\
\hline
\end{tabular}

При анализе полученных значений в табл. 3, видно, что при разбиении исходной матрицы на slice-матрицы потери информации составляют от 93,9 до 96 процентов, однако на такой же процентный показатель уменьшается количество сравнений. Таким образом альтернативный подход расчета ско- рости заполнения и потери информации также подходит для решения выше указанных оптимизационных задач. Построим график с 3D поверхностью (рис. 2).

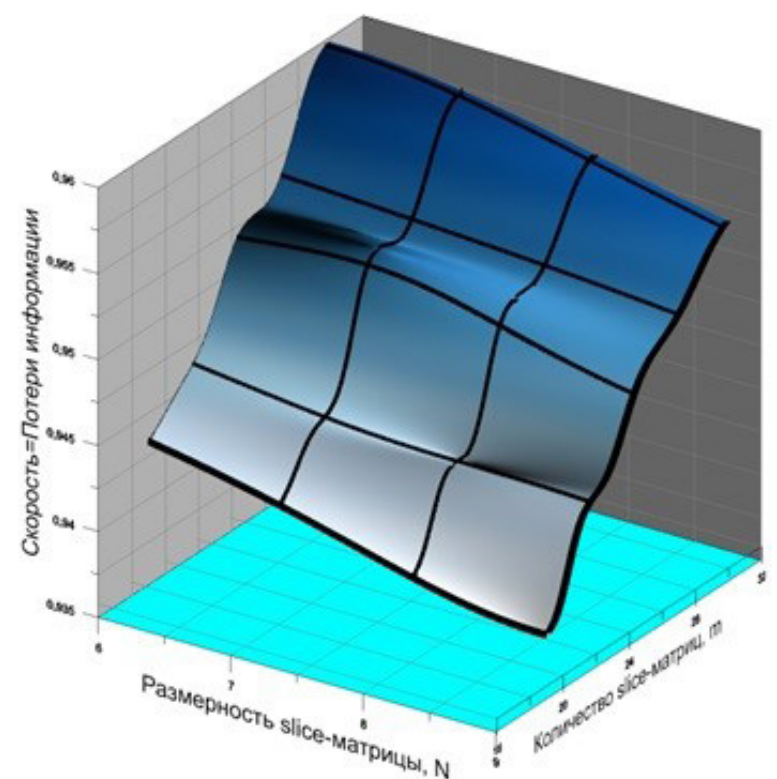

Рис. 2. Альтернативный вариант зависимости скорости (Потери инбормации) от количества и размерности slice-матрии, [Fig. 2. An alternative variant of the dependence of speed (loss of information) on the number and dimension of slice matrices]

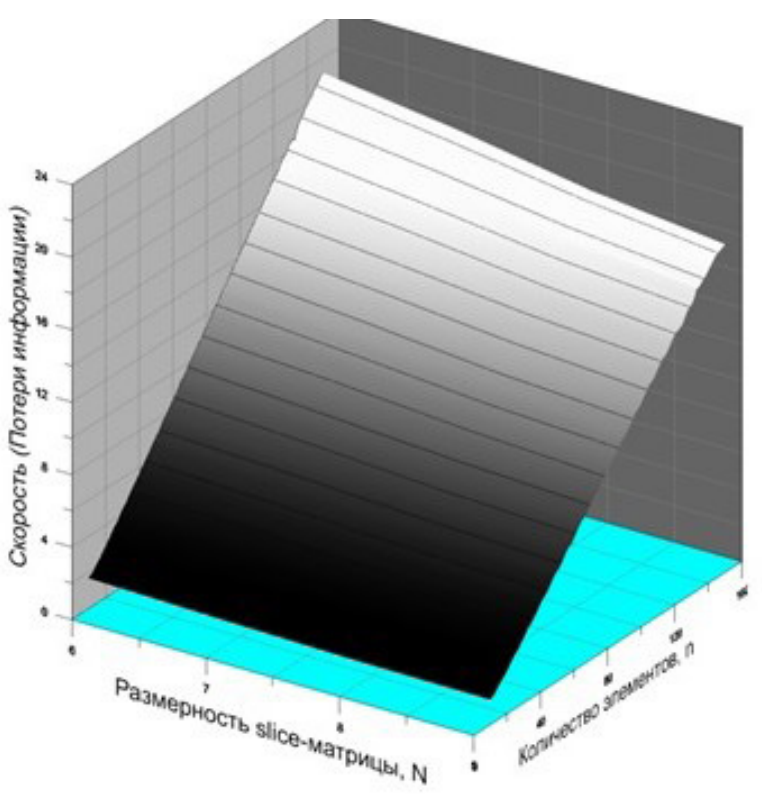

Рис. 3. График зависимости скорости (nотери информации) для разных значений $n$ и $N$ [Fig. 3. Graph of speed dependence (information loss) for different values of $n$ and $N$ ] 
Системный анализ и синтез предиктивной системь управления проиессом термоокислительной ...

На основании проведенных расчетов, результаты которых приведены в табл. 1-3 следует, что при использовании slice-матрицы размерностью 9, получаются минимальные потери информации, и решается задача 1.

Построим трехмерный график изменения скорости $C$ (потери информации П) для разного числа сравниваемых объектов $\mathrm{n}$ в интервале от 10 до 150 (рис. 3), в целях улучшения восприятия дискретной сетки значений, изобразим её с помощью 3D поверхности.

\section{ЗАКЛЮЧЕНИЕ}

Таким образом построенные графики на рис. 1-3 и выражения (10)-(15) позволяют решить оптимизационные задачи. Применение алгоритма получения вектора приоритетов позволяет использовать его в работе ситуационных центров органов внутренних дел, что позволяет более эффективно решать задачи по защите жизни, здоровья, прав и свобод граждан, противодействию преступности, охране общественного порядка, собственности и обеспечению общественной безопасности.

\section{КОНФЛИКТ ИНТЕРЕСОВ}

Авторы декларируют отсутствие явных и потенциальных конфликтов интересов, связанных с публикацией настоящей статьи.

\section{СПИСОК ЛИТЕРАТУРЫ}

1. Situation center of the $M$ inistry of internal Affairs of Russia: P rospects for development : Journal. The Police Of Russia. - 2019. - P. 26-31.
2. Ilin N. I., Demidov N. N., Novikova E. V. Situation centers. Experience, state, development dtends // Moscow. MediaPress, 2011. - 336 p.

3. Saaty T. L. Decision making with dependence and feedback: The analytic network process. Translation from English / Sceintific editor A. V. Andreichikov, O. N. Andreichikova. // 3rd Edition - Moscow. Book house «LIBROKOM», 2011. - 360 p.

4. Pyankov O. V. Development of a numerical method for determining the weights of conflict interactions / O. V. Pyankov, A. A. Terentyev // Bulletin of the Voronezh Institute of the Ministry of internal Affairs of Russia. - 2019. - No 1. P. 69-74.

5. Terentyev A. A. Development of information technology for processing expert assessments / A. A. Terentyev // Information technologies in modeling and management: Approaches, methods, solutions. - 2019. - Part 2. - P. 291-297.

6. Terentyev A. A. Calculation of the priority vector using slice-matrices / Federal service for intellectual property (ROSPATENT), Moscow. Certificate of state registration of the computer program No 2019615980.

7. Golubinsky A. N. radio Engineering systems: textbook. Manual / A. N. Golubinsky, A. A. Zibrov. - Voronezh : Voronezh Institute of the Ministry of internal Affairs of Russia, 2016. $244 \mathrm{p}$.

8. Kogan I. M. Applied Information Theory. Moscow: Radio and Communications, 1981. $216 \mathrm{p}$.

Пьянков Олег Викторович - д-р техн. наук, доц., заместитель начальника кафедры инфокоммуникационных систем и технологий.

E-mail: ovpyankov@mail.ru

ORCID iD: https://orcid.org/0000-0001-5602-7737

Терентьев Александр Андреевич - инженер кафедры инфокоммуникационных систем и технологий.

E-mail: Alextt02021993@yandex.ru

ORCID iD: https://orcid.org/0000-0002-4034-3628 


\title{
MODELLING AND PARAMETRIC OPTIMISATION OF AN EXPERT SURVEY USING SLICE MATRICES
}

\author{
(C) 2020 O. V. Pyankov, A. A. Terentyev \\ Voronezh Institute of the Ministry of the Interior of Russia \\ 53, Patriotov Avenue, 394065 Voronezh, Russian Federation
}

\begin{abstract}
Annotation. The article considers the creation and application of situation centers at internal affairs bodies under conditions of increasing complexity and heterogeneity of threats arising in modern society. The need to solve diverse tasks in order to ensure public safety and protect public order requires the engagement of experts. The article deals with the problem of conducting and processing the results of an expert survey of pairwise comparison of alternatives in the situation centers of internal affairs bodies using a previously developed algorithm based on slice matrices. We suggest that the parameters for optimizing the pairwise comparison should be the choice between speed and accuracy, depending on both the number of objects being compared by one expert and the total number of alternatives being compared. The problems relating to the parametric optimization of the expert survey were formulated. Using information theory and wellknown statements about the amount of information and the entropy, we developed an approach that allows taking into account the information losses occurring when forming slice matrices for an individual expert. In order to reduce the number of pairwise comparisons in relation to the total number of pairs of alternatives, we developed an approach that allows evaluating the gain in the speed of conducting an expert survey using slice matrices. A generalized conclusion is made about the numerical equality of speed and information loss when using slice matrices. The article presents the results of a computational experiment carried out to determine the speed and loss of information when conducting an expert survey using slice-matrices. In addition, an example of the dependence of the optimization parameters on the total number of compared alternatives and the dimensionality of the slice matrices is given. A conclusion is made about the choice of the optimal value of the parameters for conducting an expert survey.
\end{abstract}

Keywords: situation center, amount of information, entropy, parametric optimization.

\section{CONFLICT OF INTEREST}

The authors declare the absence of obvious and potential conflicts of interest related to the publication of this article.

\section{REFERENCES}

1. Situation center of the M inistry of internal Affairs of Russia: P rospects for development : Journal. The Police Of Russia. - 2019. - P. 26-31.

2. Ilin N. I., Demidov N. N., Novikova E. V. Situation centers. Experience, state, development dtends // Moscow. MediaPress, 2011. - 336 p.

Terentyev Alexander A.

e-mail: Alextt02021993@yandex.ru
3. Saaty T. L. Decision making with dependence and feedback: The analytic network process. Translation from English / Sceintific editor A. V. Andreichikov, O. N. Andreichikova. // 3rd Edition - Moscow. Book house «LIBROKOM», 2011. - 360 p.

4. Pyankov O. V. Development of a numerical method for determining the weights of conflict interactions / O. V. Pyankov, A. A. Terentyev // Bulletin of the Voronezh Institute of the Ministry of internal Affairs of Russia. - 2019. - No 1. P. 69-74.

5. Terentyev A. A. Development of information technology for processing expert assessments / A. A. Terentyev // Information technologies in modeling and management: Approaches, methods, solutions. - 2019. - Part 2. - P. 291-297. 
Системный анализ и синтез предиктивной системы управления процессом термоокислительной ...

6. Terentyev A. A. Calculation of the priority vector using slice-matrices / Federal service for intellectual property (ROSPATENT), Moscow. Certificate of state registration of the computer program No 2019615980.

7. Golubinsky A. N. Radio Engineering systems: textbook. Manual / A. N. Golubinsky,
A. A. Zibrov. - Voronezh : Voronezh Institute of the Ministry of internal Affairs of Russia, 2016. $244 \mathrm{p}$.

8. Kogan I. M. Applied Information Theory. Moscow: Radio and Communications, 1981. $216 \mathrm{p}$.

Pyankov Oleg V. - DSc in Technical Sciences, Associate Professor, Deputy Head of the Department of Information and Communication Systems and Technologies.

E-mail: ovpyankov@mail.ru

ORCID iD: https://orcid.org/0000-0001-5602-7737

Terentyev Alexander A. - Engineer, Department of Information and Communication Systems and Technologies.

E-mail: Alextt02021993@yandex.ru

ORCID iD: https://orcid.org/0000-0002-4034-3628 